\title{
Evaluation of Doping in GaP Core-Shell Nanowire pn Junction by Off-Axis Electron Holography
}

\author{
S. Yazdi ${ }^{1}$, A. Berg ${ }^{2}$, T. Kasama ${ }^{1}$, M. Beleggia ${ }^{1}$, M. T. Borgström ${ }^{2}$ and J.B. Wagner ${ }^{1}$ \\ ${ }^{1}$ Center for Electron Nanoscopy, Technical University of Denmark, DK 2800 Kgs. Lyngby, Denmark \\ 2 Solid State Physics, Lund University, Box 118, S-221 00 Lund, Sweden
}

Semiconductor nanowires (NWs) are very promising building blocks for future electronic and optoelectronic devices. Realizing this, nevertheless, requires overcoming several important challenges, such as control and evaluation of doping levels in NWs. Due to the nanoscale nature of NWs, characterization techniques, such as Hall effect measurements and four-point probes, used conventionally for the evaluation of doping in bulk materials cannot be easily applied. A quantitative technique with high spatial resolution is required for mapping the dopant levels in NWs. Here, in order to evaluate the incorporation of $\mathrm{Sn}$ as an n-type dopant in GaP core-shell nanowire $p n$ junctions, we use off-axis electron holography in the transmission electron microscope (TEM).

The GaP nanowires were grown by metal-organic vapour phase epitaxy (MOVPE) using $\mathrm{TMGa} \mathrm{PH}_{3}$, $\mathrm{HCl}, \mathrm{DEZn}$, and TESn as precursor sources and $\mathrm{H} 2$ as carrier gas. The nanowires core was grown by the vapor-liquid-solid growth mechanism on a GaP substrate, using patterned Au seeds. For $p$-type doping of the core, DEZn with the molar fraction of $\chi_{\mathrm{DEZn}}=9.3 \times 10^{-6}$ was used. After growing the nanowire core the Au seeds were removed by wet etching before growing the shell. Using different molar fraction of TESn during the growth of the n-type shell, we prepared five nanowire samples with identical dopant concentration in their $p$-type cores and different dopant concentration in their $n$-type shells.

A cross-sectional TEM specimen was prepared from each nanowire sample using focused ion beam milling (FIB) in an FEI Helios DualBeam system. The same milling conditions, $30 \mathrm{keV}$ beam in the thinning and 2 $\mathrm{keV}$ beam in the final polishing, were used in preparing all the TEM specimens. By using the configuration shown in Fig. 1a in our DualBeam system, it was possible to measure the current-voltage (IV) characteristic of a specific single nanowire before preparing its TEM specimen. A typical IV curve measured from a single GaP nanowire is shown in Fig. 1a, confirming the presence of a pn junction.

Holograms were acquired from each specimen using an FEI Titan 80-300ST TEM operated at $120 \mathrm{kV}$. From the reconstructed phase images, such as the one shown in Fig. 2, the built-in potential across the core-shell $p n$ junction in each nanowire sample was calculated taking the crystalline thickness into account. Here, it can be assumed that the effects that FIB-damage and electron beam illumination might have on the measurements of the built-in potentials are the same for all the nanowire samples as the conditions for FIB-preparation and electron hologram acquisition were the same for all samples. The main result is shown in Fig. 3 where the measured built-in potentials across the core-shell pn junctions are plotted as a function of TESn molar fraction. It is clear that the incorporation of $\mathrm{Sn}$ atoms as an $n$-type dopant in the nanowires shell increases linearly with the TESn molar fraction within the examined range. This investigation also shows that off-axis electron holography is a powerful tool for the evaluation of doping processes in nanowires.

\section{References}

[1] The authors acknowledge funding from 'Nanowires for solid state lighting'. NMP.2011.2.2-3, Project ID: 280773 


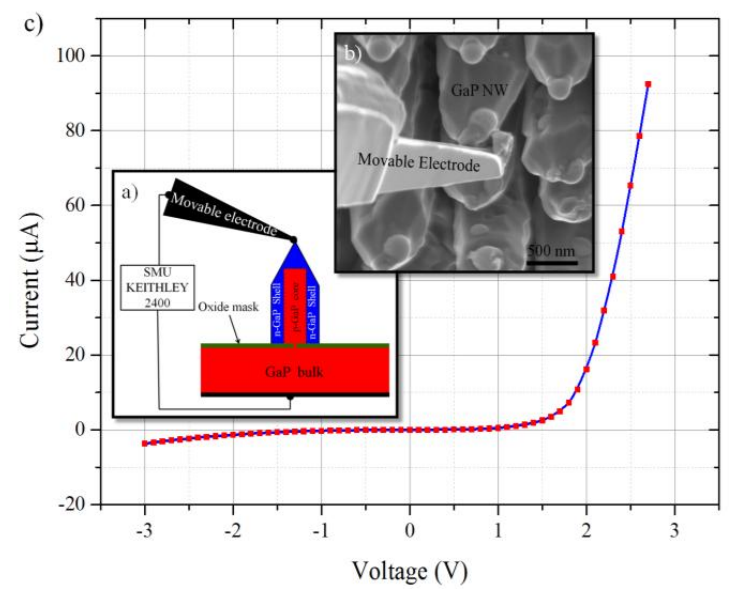

Figure 2. a) A typical reconstructed phase image and b) the corresponding thickness map calculated from the reconstructed amplitude image. c) The phase and thickness profile from the same region marked with an arrow in (a) and (b). In all of the measurements, the thickness maps were used for checking the uniformity of the specimen thicknesses and for reassuring that the diffraction contrast has been suppressed appropriately by tilting the specimen. For calculating the built-in potentials, $\mathrm{V}_{\mathrm{bi}}$, the phase steps, $\Delta \varphi$, were measured across the core-shell junctions as shown in (c) and then divided by the crystalline thickness of the specimens, $\mathrm{t}$, and constant $\mathrm{C}_{\mathrm{E}}\left(\mathrm{V}_{\mathrm{bi}}=\Delta \varphi / \mathrm{C}_{\mathrm{E}} \mathrm{t}\right)$. In this calculation, no crystalline inactive layer was considered.

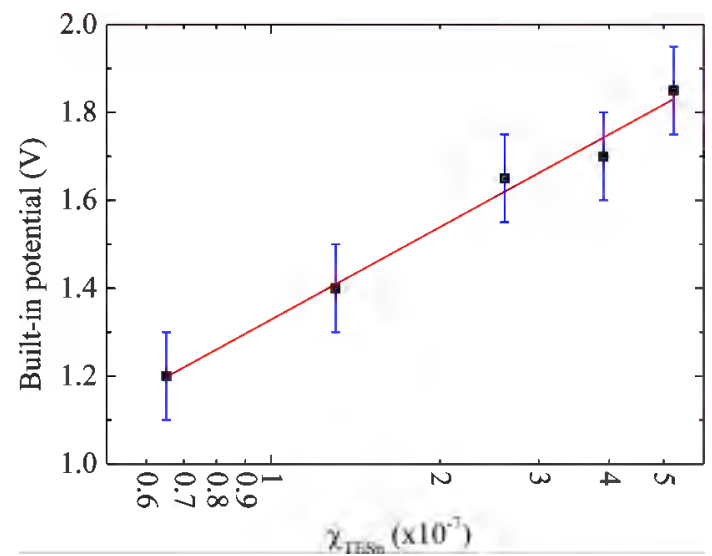

Figure 1. a) Schematic diagram showing how IV curve was measured from single core-shell nanowire $p n$ junction in a DualBeam system. The electrical contact to the nanowires core was made through a large fix contact at the bottom of the sample. The micromanipulator in the DualBeam system was used as a movable electrode for making contacts to the shell of single nanowires. b) SEM image of a $\mathrm{GaP}$ nanowire at $45^{\circ}$ tilt angle in contact with the micromanipulator. Sn-rich particles can be seen on the nanowires surfaces. c) A typical IV curve measured from the biasing configuration in a).
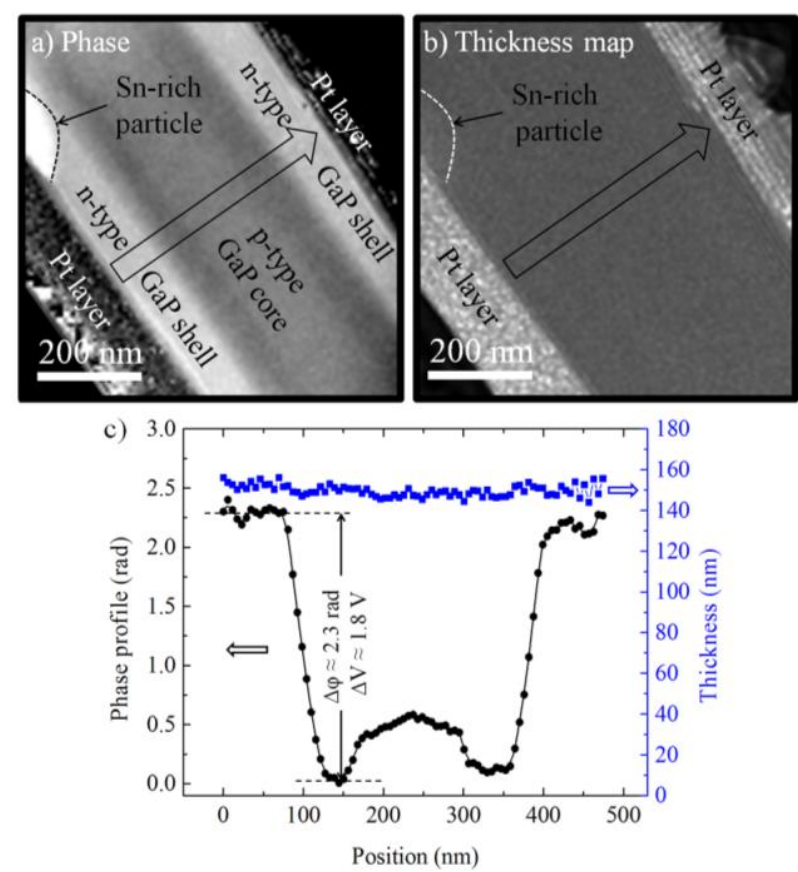

Figure 3. Measured built-in potentials across the GaP core-shell $p n$ junctions by off-axis electron holography as a function of TESn molar fraction used for doping the nanowires shell. This plot shows a linear relationship between the TESn molar fraction and dopant concentration. Note that the horizontal axis is in logarithmic scale. 